\title{
On a Certain Extension of the Hurwitz-Lerch Zeta Function
}

Rakesh K. Parmar and R. K. Raina

\begin{abstract}
Our purpose in this paper is to consider a generalized form of the extended Hurwitz-Lerch Zeta function. For this extended Hurwitz-Lerch Zeta function, we obtain some classical properties which includes various integral representations, a differential formula, Mellin transforms and certain generating relations. We further consider an application to probability distributions and also point out some important special cases of the main results.
\end{abstract}

AMS Subject Classification (2000). Primary 11M06, 11M35, 33B15; Secondary 11B68, 33C05, 33C65, 33C90

Keywords. Generalized Hurwitz-Lerch Zeta function; Extended Beta function; Extended hypergeometric function; Extended Hurwitz-Lerch Zeta function; Mellin Transform; Probability density function

\section{Introduction}

Let $\mathbb{N}, \mathbb{Z}^{-}, \mathbb{R}, \mathbb{R}^{+}$and $\mathbb{C}$ denote the sets of positive integers, negative integers, real numbers, positive real numbers and complex numbers, respectively, and also, let $\mathbb{N}_{0}:=\mathbb{N} \cup\{0\}$ and $\mathbb{Z}_{0}^{-}:=\mathbb{Z}^{-} \cup\{0\}$.

The Hurwitz-Lerch Zeta function $\Phi(z, s, a)$ is defined by (see, e.g., [5, p. 
27, Eq. 1.11(1)]; see also [11, p. 121] and [12, p. 194]):

$$
\Phi(z, s, a):=\sum_{n=0}^{\infty} \frac{z^{n}}{(n+a)^{s}}
$$

$\left(a \in \mathbb{C} \backslash \mathbb{Z}_{0}^{-} ; s \in \mathbb{C} \quad\right.$ when $\quad|z|<1 ; \Re(s)>1$ when $\left.|z|=1\right)$.

Also, the integral representation of $\Phi(z, s, a)$ (see, e.g., [5, p. 27, Eq. $1.11(3)]$; see also [12, p. 194, Eq. 2.5(4)]) is given by

$$
\Phi(z, s, a)=\frac{1}{\Gamma(s)} \int_{0}^{\infty} \frac{t^{s-1} e^{-a t}}{1-z e^{-t}} d t=\frac{1}{\Gamma(s)} \int_{0}^{\infty} \frac{t^{s-1} e^{-(a-1) t}}{e^{t}-z} d t
$$

$(\Re(a)>0 ; \Re(s)>0 \quad$ when $|z| \leqq 1(z \neq 1) ; \Re(s)>1 \quad$ when $\quad z=1)$.

Certain forms of generalizations of the Hurwitz-Lerch Zeta function $\Phi(z, s, a)$ have been considered by various authors, see, e.g., [3-7]). Goyal and Laddha [8, p. 100, Eq. (1.5)] and Garg et al. [7, p. 313, Eq. (1.7)] studied certain functions which are, respectively, defined by

$$
\Phi_{\mu}^{*}(z, s, a):=\sum_{n=0}^{\infty} \frac{(\mu)_{n}}{n !} \frac{z^{n}}{(n+a)^{s}}
$$

$\left(\mu \in \mathbb{C} ; a \in \mathbb{C} \backslash \mathbb{Z}_{0}^{-} ; s \in \mathbb{C} \quad\right.$ when $\quad|z|<1 ; \Re(s-\mu)>1 \quad$ when $\left.\quad|z|=1\right)$ and

$$
\Phi_{\lambda, \mu ; \nu}(z, s, a):=\sum_{n=0}^{\infty} \frac{(\lambda)_{n}(\mu)_{n}}{(\nu)_{n} n !} \frac{z^{n}}{(n+a)^{s}}
$$

$\left(\lambda, \mu \in \mathbb{C} ; \nu, a \in \mathbb{C} \backslash \mathbb{Z}_{0}^{-} ; s \in \mathbb{C} \quad\right.$ when $|z|<1 ; \Re(s+\nu-\lambda-\mu)>1$ when $|z|=1$ ), where $(\lambda)_{\nu}$ denotes the usual Pochhammer symbol (for $\lambda \in \mathbb{C}$ ) defined by (see [12, p. 2 and p. 5]):

$$
\begin{aligned}
(\lambda)_{\nu}: & = \begin{cases}1 & (n=0) \\
\lambda(\lambda+1) \ldots(\lambda+\nu-1) & (\nu \in \mathbb{N})\end{cases} \\
& =\frac{\Gamma(\lambda+\nu)}{\Gamma(\lambda)} \quad\left(\lambda \in \mathbb{C} \backslash \mathbb{Z}_{0}^{-}\right),
\end{aligned}
$$

where $\Gamma(\lambda)$ is the familiar (Euler's) Gamma function (see, e.g., [12, Chapter $1])$. 
The corresponding integral representations of (1.3) and (1.4) are given, respectively, by

$$
\begin{gathered}
\Phi_{\mu}^{*}(z, s, a)=\frac{1}{\Gamma(s)} \int_{0}^{\infty} \frac{t^{s-1} e^{-a t}}{\left(1-z e^{-t}\right)^{\mu}} d t=\frac{1}{\Gamma(s)} \int_{0}^{\infty} \frac{t^{s-1} e^{-(a-1) t}}{\left(e^{t}-z\right)^{\mu}} d t \\
(\Re(a)>0 ; \Re(s)>0 \quad \text { when } \quad|z| \leqq 1(z \neq 1) ; \Re(s)>1 \quad \text { when } \quad z=1)
\end{gathered}
$$

and

$$
\Phi_{\lambda, \mu ; \nu}(z, s, a):=\frac{1}{\Gamma(s)} \int_{0}^{\infty} t^{s-1} e^{-a t}{ }_{2} F_{1}\left(\lambda, \mu ; \nu ; z e^{-t}\right) d t
$$

$(\Re(a)>0 ; \Re(s)>0$ when $|z| \leqq 1(z \neq 1) ; \Re(s)>1$ when $z=1)$.

In their paper, Chaudhry et al. [1] presented the following extension of the Beta function:

$$
B(x, y ; p)=\int_{0}^{1} t^{x-1}(1-t)^{y-1} \exp \left(-\frac{p}{t(1-t)}\right) d t \quad(\Re(p)>0),
$$

and showed that this extension has certain connections with the Macdonald, Error, and Whittaker functions.

Subsequently, Chaudhry et al. [2] used the above function $B(x, y ; p)$ to provide an extension of the hypergeometric function which is given by

$$
\begin{gathered}
F_{p}(a, b ; c ; z)=\sum_{n=0}^{\infty}(a)_{n} \frac{B(b+n, c-b ; p)}{B(b, c-b)} \frac{z^{n}}{n !} \\
(p \geqq 0,|z|<1 ; \Re(c)>\Re(b)>0) .
\end{gathered}
$$

Evidently, the special cases of (1.8) and (1.9) when $p=0$ reduce immediately to the classical Eulerian Beta and Gauss's hypergeometric function, respectively.

Motivated by these aforecited cosiderations, we first give an extension of the generalized Hurwitz-Lerch Zeta function (1.4). We then investigate certain properties of the extended generalized Hurwitz-Lerch Zeta function and derive for this class of functions various integral representations, a derivative formula, Mellin transform and certain generating relations. An application to probability distributions is also considered and we also point out some special cases of the main results. 


\section{Extended Generalized Hurwitz-Lerch Zeta Function}

In terms of the extended Beta function $B(x, y ; p)$ defined by $(1.8)$, we propose a new extension of generalized Hurwitz-Lerch Zeta function by changing

$$
\frac{(\mu)_{n}}{(\nu)_{n}}=\frac{B(\mu+n, \nu-\mu)}{B(\mu, \nu-\mu)} \rightarrow \frac{B(\mu+n, \nu-\mu ; p)}{B(\mu, \nu-\mu)}
$$

in (1.4) and thus, define this new extended form as follows:

$$
\Phi_{\lambda, \mu ; \nu}(z, s, a ; p):=\sum_{n=0}^{\infty} \frac{(\lambda)_{n}}{n !} \frac{B(\mu+n, \nu-\mu ; p)}{B(\mu, \nu-\mu)} \frac{z^{n}}{(n+a)^{s}}
$$

$\left(p \geqq 0 ; \lambda, \mu \in \mathbb{C} ; \nu, a \in \mathbb{C} \backslash \mathbb{Z}_{0}^{-} ; s \in \mathbb{C}\right.$ when $|z|<1 ; \Re(s+\nu-\lambda-\mu)>1$ when $|z|=1)$.

Remark 2.1. The following special or limit cases of the generalized HurwitzLerch Zeta function $\Phi_{\lambda, \mu ; \nu}(z, s, a ; p)$ defined by (2.1) are worth noting.

Case 1. If we set $\lambda=1$, then (2.1) yields the following special case of another extended form of the generalized Hurwitz-Lerch Zeta function $\Phi_{\mu ; \nu}^{1,1}(z, s, a ; p)$ studied earlier by Lin and Srivastava [9, p. 727, Eq. (8)] (with $\rho=\sigma=1)$ :

$$
\Phi_{\mu ; \nu}^{1,1}(z, s, a ; p):=\Phi_{1, \mu ; \nu}(z, s, a ; p):=\sum_{n=0}^{\infty} \frac{B(\mu+n, \nu-\mu ; p)}{B(\mu, \nu-\mu)} \frac{z^{n}}{(n+a)^{s}}
$$

$\left(p \geqq 0 ; \mu \in \mathbb{C} ; \nu, a \in \mathbb{C} \backslash \mathbb{Z}_{0}^{-} ; s \in \mathbb{C}\right.$ when $|z|<1 ; \Re(s+\nu-\mu)>1$ when $|z|=1)$.

Case 2. Upon setting $\lambda=\nu=1$ in (2.1), we have the extended generalized Hurwitz-Lerch Zeta function $\Phi_{\mu}^{*}(z, s, a ; p)$ of Goyal and Laddha [8] in the following form:

$$
\begin{aligned}
& \Phi_{\mu}^{*}(z, s, a ; p):=\Phi_{1, \mu ; 1}(z, s, a ; p):=\sum_{n=0}^{\infty} \frac{B(\mu+n, 1-\mu ; p)}{B(\mu, 1-\mu)} \frac{z^{n}}{(n+a)^{s}} \\
& \left(p \geqq 0 ; \mu \in \mathbb{C} ; a \in \mathbb{C} \backslash \mathbb{Z}_{0}^{-} ; s \in \mathbb{C} \text { when }|z|<1 ; \Re(s+1-\mu)>1\right. \text { when } \\
& |z|=1) .
\end{aligned}
$$


Case 3. A limit case of the generalized Hurwitz-Lerch function $\Phi_{\lambda, \mu ; \nu}(z, s, a$; $p$ ) defined by (2.1) (which is particularly of interest in our present investigation) is given by

$\Phi_{\mu ; \nu}^{*}(z, s, a ; p):=\lim _{|\lambda| \rightarrow \infty}\left\{\Phi_{\lambda, \mu ; \nu}\left(\frac{z}{\lambda}, s, a ; p\right)\right\}:=\sum_{n=0}^{\infty} \frac{B(\mu+n, \nu-\mu ; p)}{B(\mu, \nu-\mu)} \frac{z^{n}}{n !(n+a)^{s}}$

$\left(p \geqq 0 ; \mu \in \mathbb{C} ; \nu, a \in \mathbb{C} \backslash \mathbb{Z}_{0}^{-} ; s \in \mathbb{C}\right.$ when $|z|<1 ; \Re(s+\nu-\mu)>1$ when $|z|=1)$.

Remark 2.2. The special cases of (2.1) and (2.3) when $p=0$ are easily seen to reduce to (1.4) and (1.3), respectively, in view of the following relationships of the functions (defined above):

$$
\Phi_{\lambda, \mu ; \nu}(z, s, a ; 0)=\Phi_{\lambda, \mu ; \nu}(z, s, a) \quad \text { and } \quad \Phi_{1, \mu ; 1}(z, s, a ; 0)=\Phi_{\mu}^{*}(z, s, a),
$$

Also, for $p=0$ in (2.2), we have the following special case of the generalized Hurwitz-Lerch function of Lin and Srivastava [9, p. 727, Eq. (8)] (with $\rho=\sigma=1)$ :

$$
\Phi_{1, \mu ; \mu}(z, s, a ; 0)=\Phi_{\mu ; \nu}^{1,1}(z, s, a) .
$$

\section{$3 \quad$ Integral Representations and Derivative Formula}

In this section, we present certain integral representations and a derivative formula for the extended Hurwitz-Lerch Zeta function defined by (2.1).

Theorem 3.1. The following integral representation holds true:

$$
\begin{aligned}
& \Phi_{\lambda, \mu ; \nu}(z, s, a ; p):=\frac{1}{\Gamma(s)} \int_{0}^{\infty} t^{s-1} e^{-a t} F_{p}\left(\lambda, \mu ; \nu ; z e^{-t}\right) d t \\
& (p \geqq 0 ; \Re(a)>0 ; \Re(s)>0 \text { when }|z| \leqq 1(z \neq 1) ; \Re(s)>1 \text { when } \\
& z=1) .
\end{aligned}
$$

Proof. Using the following Eulerian integral:

$$
\frac{1}{(n+a)^{s}}:=\frac{1}{\Gamma(s)} \int_{0}^{\infty} t^{s-1} e^{-(n+a) t} d t \quad\left(\min \{\Re(s), \Re(a)\}>0 ; n \in \mathbb{N}_{0}\right)
$$


in (2.1), we have

$$
\Phi_{\lambda, \mu ; \nu}(z, s, a ; p)=\sum_{n=0}^{\infty} \frac{(\lambda)_{n}}{n !} \frac{B(\mu+n, \nu-\mu ; p)}{B(\mu, \nu-\mu)}\left(\frac{1}{\Gamma(s)} \int_{0}^{\infty} t^{s-1} e^{-(n+a) t} d t\right) .
$$

Interchanging the order of summation and integration under the condition stated in Theorem 3.1, we get

$$
\Phi_{\lambda, \mu ; \nu,}(z, s, a ; p):=\frac{1}{\Gamma(s)} \int_{0}^{\infty} t^{s-1} e^{-a t}\left(\sum_{n=0}^{\infty}(\lambda)_{n} \frac{B(\mu+n, \nu-\mu ; p)}{B(\mu, \nu-\mu)} \frac{\left(z e^{-t}\right)^{n}}{n !}\right) d t .
$$

Now using the definition (1.9) to express the involved sum as an extended hypergeometric function, we are led to the desired result.

Theorem 3.2. The following integral representations hold true:

$$
\begin{gathered}
\Phi_{\lambda, \mu ; \nu}(z, s, a ; p)=\frac{e^{-2 p} \Gamma(\nu)}{\Gamma(\mu) \Gamma(\nu-\mu)} \int_{0}^{\infty} \frac{x^{\mu-1}}{(1+x)^{\nu}} \exp \left(-p\left(x+\frac{1}{x}\right)\right) \Phi_{\lambda}^{*} \\
\left(\frac{z x}{1+x}, s, a\right) d x \\
(p \geqq 0 ; \Re(\nu)>\Re(\mu)>0)
\end{gathered}
$$

and

$$
\begin{gathered}
\Phi_{\lambda, \mu ; \nu}(z, s, a ; p)=\frac{e^{-2 p} \Gamma(\nu)}{\Gamma(s) \Gamma(\mu) \Gamma(\nu-\mu)} \\
\times \int_{0}^{\infty} \int_{0}^{\infty} \frac{t^{s-1} e^{-a t} x^{\mu-1}}{(1+x)^{\nu}} \exp \left(-p\left(x+\frac{1}{x}\right)\right)\left(1-\frac{z x e^{-t}}{1+x}\right)^{-\lambda} d t d x \\
(p \geqq 0 ; \Re(\nu)>\Re(\mu)>0, \min \{\Re(s), \Re(a)\}>0),
\end{gathered}
$$

provided that the integrals in the right-hand sides of the assertions (3.3) and (3.4) converge.

Proof. By setting $\alpha=\mu+n$ and $\beta=\nu-\mu$ in the following integral representation of the extended Beta-function (see, e.g.,[1, p. 22, Equation (2.8)]):

$$
B(\alpha, \beta ; p)=e^{-2 p} \int_{0}^{\infty} \frac{x^{\alpha-1}}{(1+x)^{\alpha+\beta}} \exp \left(-p\left(x+\frac{1}{x}\right)\right) d x \quad(\Re(p)>0),
$$




$$
(p \geqq 0 ; \Re(\alpha)>0, \Re(\beta)>0)
$$

we find that

$$
B(\mu+n, \nu-\mu ; p)=e^{-2 p} \int_{0}^{\infty} \frac{x^{\mu+n-1}}{(1+x)^{\nu+n}} \exp \left(-p\left(x+\frac{1}{x}\right)\right) d x
$$

which by appealing to the (2.1) and using (1.3) immediately yields the first assertion (3.3) of Theorem 3.2.

Also, in view of (3.1) and (3.6), we also obtain

$$
\begin{aligned}
& \Phi_{\lambda, \mu ; \nu}(z, s, a ; p):=\frac{1}{\Gamma(s)} \int_{0}^{\infty} t^{s-1} e^{-a t} \sum_{n=0}^{\infty}(\lambda)_{n} \frac{B(\mu+n, \nu-\mu ; p)}{B(\mu, \nu-\mu)} \frac{\left(z e^{-t}\right)^{n}}{n !} d t \\
& =\frac{e^{-2 p} \Gamma(\nu)}{\Gamma(s) \Gamma(\mu) \Gamma(\nu-\mu)} \int_{0}^{\infty} \int_{0}^{\infty} \frac{t^{s-1} e^{-a t} x^{\mu-1}}{(1+x)^{\nu}} \exp \left(-p\left(x+\frac{1}{x}\right)\right) \\
& \quad \sum_{n=0}^{\infty} \frac{(\lambda)_{n}}{n !}\left(\frac{z x e^{-t}}{1+x}\right)^{n} d t d x
\end{aligned}
$$

which in view of the binomial expansion

$$
(1-z t)^{-a}=\sum_{n=0}^{\infty}(a)_{n} \frac{(z t)^{n}}{n !}
$$

leads us to the second assertion (3.4) of Theorem 3.2.

Theorem 3.3. The following integral representation holds true:

$$
\Phi_{\lambda, \mu ; \nu}(z, s, a ; p):=\frac{1}{\Gamma(\lambda)} \int_{0}^{\infty} t^{\lambda-1} e^{-t} \Phi_{\mu ; \nu}^{*}(z t, s, a ; p) d t
$$

$(p \geqq 0 ; \Re(\lambda)>0, \Re(a)>0 ; \Re(s)>0$ when $|z| \leqq 1(z \neq 1) ; \Re(s)>1$ when $z=1)$, where $\Phi_{\mu ; \nu}^{*}(z, s, a ; p)$ is the limiting case defined by $(2.4)$.

Proof. Using the integral representation of the Pochhammer symbol $(\lambda)_{n}$ :

$$
(\lambda)_{n}=\frac{1}{\Gamma(\lambda)} \int_{0}^{\infty} t^{\lambda+n-1} e^{-t} d t
$$

in (2.1) and inverting the order of summation and integration (which is permissible under the conditions stated with Theorem 3.3), we get

$$
\Phi_{\lambda, \mu ; \nu}(z, s, a ; p)=\frac{1}{\Gamma(\lambda)} \int_{0}^{\infty} t^{\lambda-1} e^{-t} \sum_{n=0}^{\infty} \frac{B(\mu+n, \nu-\mu ; p)}{B(\mu, \nu-\mu)} \frac{(z t)^{n}}{n !(n+a)^{s}} d t .
$$

Applying now (2.4), we get the desired integral representation. 
We next derive the following derivative formula for the function $\Phi_{\lambda, \mu ; \nu}(z, s, a ; p)$ defined by (2.1).

Theorem 3.4. The following derivative formula for $\Phi_{\lambda, \mu ; \nu}(z, s, a ; p)$ holds true:

$$
\frac{d^{n}}{d z^{n}}\left\{\Phi_{\lambda, \mu ; \nu}(z, s, a ; p)\right\}=\frac{(\lambda)_{n}(\mu)_{n}}{(\nu)_{n}} \Phi_{\lambda+n, \mu+n ; \nu+n}(z, s, a+n ; p) \quad\left(n \in \mathbb{N}_{0}\right) .
$$

Proof. Differentiating (2.1) with respect to $z$, we obtain

$$
\frac{d}{d z}\left\{\Phi_{\lambda, \mu ; \nu}(z, s, a ; p)\right\}=\sum_{n=1}^{\infty} \frac{(\lambda)_{n}}{(n-1) !} \frac{B(\mu+n, \nu-\mu ; p)}{B(\mu, \nu-\mu)} \frac{z^{n-1}}{(n+a)^{s}} .
$$

which upon replacing $n$ by $n+1$ in the right-hand side of (3.11), and using the identities that

$$
B(\mu, \nu-\mu)=\frac{\nu}{\mu} B(\mu+1, \nu-\mu) \quad \text { and } \quad(a)_{n+1}=a(a+1)_{n}
$$

gives the derivative formula that

$$
\frac{d}{d z}\left\{\Phi_{\lambda, \mu ; \nu}(z, s, a ; p)\right\}=\frac{\lambda \mu}{\nu} \Phi_{\lambda+1, \mu+1 ; \nu+1}(z, s, a+1 ; p) .
$$

A repeated application of the this above procedure $n$-times gives us the general formula (3.10).

\section{Mellin Transform and Generating Relations}

In this section, we obtain Mellin transform and certain generating relations for the extended Hurwitz-Lerch Zeta function $\Phi_{\lambda, \mu ; \nu}(z, s, a ; p)$ defined by (2.1).

The Mellin transform of a suitable integrable function $f(t)$ with index $\alpha$ is defined, as usual, by

$$
\mathcal{M}\{f(\tau): \tau \rightarrow \alpha\}:=\int_{0}^{\infty} \tau^{\alpha-1} f(\tau) d \tau,
$$

provided that the improper integral in (4.1) exists. 
Theorem 4.1. The Mellin transform of the function $\Phi_{\lambda, \mu ; \nu}(z, s, a ; p)$ defined by (2.1) is given by

$$
\begin{array}{r}
\mathcal{M}\left\{\Phi_{\lambda, \mu ; \nu}(z, s, a ; p)(z, s, a): p \rightarrow \alpha\right\} \\
=\frac{\Gamma(\alpha) B(\mu+\alpha, \nu-\mu+\alpha)}{B(\mu, \nu-\mu)} \Phi_{\lambda, \mu+\alpha, \nu+2 \alpha}(z, s, a) \\
(\Re(\alpha)>0 \quad \text { and } \quad \Re(\lambda+\alpha)>0) .
\end{array}
$$

Proof. Using the definition (4.1) of the Mellin transform, we find from (2.1) that

$$
\begin{aligned}
\mathcal{M}\left\{\Phi_{\lambda, \mu ; \nu}\right. & (z, s, a ; p): p \rightarrow \alpha\}:=\int_{0}^{\infty} p^{\alpha-1} \Phi_{\lambda, \mu ; \nu}(z, s, a ; p) d p \\
& =\int_{0}^{\infty} p^{\alpha-1}\left(\sum_{n=0}^{\infty} \frac{(\lambda)_{n}}{n !} \frac{B(\mu+n, \nu-\mu ; p)}{B(\mu, \nu-\mu)} \frac{z^{n}}{(n+a)^{s}}\right) d p \\
& =\sum_{n=0}^{\infty} \frac{(\lambda)_{n}}{n !} \frac{z^{n}}{(n+a)^{s}} \frac{1}{B(\mu, \nu-\mu)} \int_{0}^{\infty} p^{\alpha-1} B(\mu+n, \nu-\mu ; p) d p .
\end{aligned}
$$

Applying the following result given by ([1, p. 21, Eq. (2.1)])

$$
\begin{gathered}
\int_{0}^{\infty} p^{\alpha-1} B(x, y ; p) d p=\Gamma(\alpha) B(x+\alpha, y+\alpha) \\
(\Re(\alpha)>0, \quad \Re(x+\alpha)>0, \quad \Re(y+\alpha)>0,
\end{gathered}
$$

we obtain

$$
\begin{aligned}
\mathcal{M}\left\{\Phi_{\lambda, \mu ; \nu}(z, s, a ; p)\right. & : p \rightarrow \alpha\}= \\
= & \Gamma(\alpha) \sum_{n=0}^{\infty} \frac{(\lambda)_{n}}{n !} \frac{z^{n}}{(n+a)^{s}} \frac{B(\mu+\alpha+n, \nu-\mu+\alpha)}{B(\mu, \nu-\mu)} \\
= & \frac{\Gamma(\alpha) B(\mu+\alpha, \nu-\mu+\alpha)}{B(\mu, \nu-\mu)} \sum_{n=0}^{\infty} \frac{(\lambda)_{n}(\mu+\alpha)_{n}}{(\nu+2 \alpha)_{n}} \frac{z^{n}}{n !(n+a)^{s}},
\end{aligned}
$$

which upon using (2.1) gives the desired Mellin transform representation. 
Remark 4.1. The special case of (4.2) when $\alpha=1$ yields the following integral of the function $\Phi_{\lambda, \mu ; \nu}(z, s, a ; p)$ with respect to the variable $p$ in terms of the generalized Hurwitz-Lerch Zeta function defined by (1.4):

$$
\int_{0}^{\infty} \Phi_{\lambda, \mu ; \nu}(z, s, a ; p) d p=\frac{\Gamma(\alpha) B(\mu+1, \nu-\mu+1)}{B(\mu, \nu-\mu)} \Phi_{\lambda, \mu+1, \nu+2}(z, s, a) .
$$

We next derive the following generating relations for the function $\Phi_{\lambda, \mu ; \nu}(z, s$, $a ; p)$ defined by (2.1).

Theorem 4.2. The following generating function for $\Phi_{\lambda, \mu ; \nu}(z, s, a ; p)$ in (2.1) holds true:

$$
\begin{gathered}
\sum_{n=0}^{\infty}(\lambda)_{n} \Phi_{\lambda+n, \mu ; \nu}(z, s, a ; p) \frac{t^{n}}{n !}=(1-t)^{-\lambda} \Phi_{\lambda+n, \mu ; \nu}\left(\frac{z}{1-t}, s, a ; p\right) \\
(p \geqq 0, \lambda \in \mathbb{C} \text { and }|t|<1) .
\end{gathered}
$$

Proof. Let the left-hand side of the assertion (4.6) be denoted by $S$. Then, from the definition (2.1), we have

$$
\begin{aligned}
S & =\sum_{n=0}^{\infty}(\lambda)_{n}\left\{\sum_{k=0}^{\infty}(\lambda+n)_{k} \frac{B(\mu+k, \nu-\mu ; p)}{B(\mu, \nu-\mu)} \frac{z^{k}}{k !(k+a)^{s}}\right\} \frac{t^{n}}{n !} \\
& =\sum_{k=0}^{\infty}(\lambda)_{k} \frac{B(\mu+k, \nu-\mu ; p)}{B(\mu, \nu-\mu)}\left\{\sum_{n=0}^{\infty}(\lambda+k)_{n} \frac{t^{n}}{n !}\right\} \frac{z^{k}}{k !(k+a)^{s}}
\end{aligned}
$$

upon reversal of the order of summation and use of the identity $(\lambda)_{n}(\lambda+n)_{k}=$ $(\lambda)_{k}(\lambda+k)_{n}$.

Using the binomial expansion

$$
(1-t)^{-\lambda-k}=\sum_{n=0}^{\infty}(\lambda+k)_{n} \frac{t^{n}}{n !} \quad(|t|<1)
$$

and interpreting in terms of $(2.1)$ as a function of the form $\Phi_{\lambda+n, \mu ; \nu}\left(\frac{z}{1-t}, s\right.$, $a ; p)$, we are thus lead to the assertion (4.6) of Theorem 4.2.

Our next result on the generating function is contained in the following: 
Theorem 4.3. The following generating function for $\Phi_{\lambda, \mu ; \nu}(z, s, a ; p)$ in $(2.1)$ holds true:

$$
\begin{gathered}
\sum_{n=0}^{\infty} \frac{(s)_{n}}{n !} \Phi_{\lambda, \mu ; \nu}(z, s+n, a ; p) t^{n}=\Phi_{\lambda, \mu ; \nu}(z, s, a-t ; p) \\
(p \geqq 0, \lambda \in \mathbb{C} \text { and }|t|<|a| ; s \neq 1) .
\end{gathered}
$$

Proof. Using (2.1) in the right-hand side of the assertion (4.7), we have

$$
\begin{aligned}
\Phi_{\lambda, \mu ; \nu} & (z, s, a-t ; p)=\sum_{k=0}^{\infty}(\lambda)_{k} \frac{B(\mu+k, \nu-\mu ; p)}{B(\mu, \nu-\mu)} \frac{z^{k}}{k !(k+a-t)^{s}} \\
= & \sum_{k=0}^{\infty}(\lambda)_{k} \frac{B(\mu+k, \nu-\mu ; p)}{B(\mu, \nu-\mu)} \frac{z^{k}}{k !(k+a)^{s}}\left(1-\frac{t}{k+a}\right)^{-s} \\
= & \sum_{k=0}^{\infty}(\lambda)_{k} \frac{B(\mu+k, \nu-\mu ; p)}{B(\mu, \nu-\mu)} \frac{z^{k}}{k !(k+a)^{s}}\left\{\sum_{n=0}^{\infty} \frac{(s)_{n}}{n !} \frac{t^{n}}{(k+a)^{n}}\right\} \\
= & \sum_{n=0}^{\infty} \frac{(s)_{n}}{n !}\left\{\sum_{k=0}^{\infty}(\lambda)_{k} \frac{B(\mu+k, \nu-\mu ; p)}{B(\mu, \nu-\mu)} \frac{z^{k}}{k !(k+a)^{s+n}}\right\} t^{n} .
\end{aligned}
$$

The desired assertion (4.7) of Theorem 4.3 follows now on making use of (2.1).

\section{Application to the Probability Distributions}

In this concluding section, we consider a general probability distribution involving the extended generalized Hurwitz-Lerch Zeta function (2.1) defined as follow:

Definition 5.1. A random variable $\xi$ is said to be the generalized Hurwitz distributed if its probability density function is given by

$$
f_{\xi}(a)=: \begin{cases}\frac{s \Phi_{\lambda, \mu ; \nu}(z, s+1, a ; p)}{\Phi_{\lambda, \mu ; \nu}(z, s, 1 ; p)}, & a \geqq 1 \\ 0 & \text { otherwise }\end{cases}
$$

since one can easily verify that

$$
\int_{1}^{\infty} f_{\xi}(a) d a=1
$$


where it is tacitly assumed that the arguments $z, s, p$ and the parameters $\lambda, \mu$ and $\nu$ are fixed and suitably constrained so that the probability density function $f_{\xi}(a)$ remains nonnegative.

Theorem 5.1. Let $\xi$ be a continuous random variable with its probability density function defined by (5.1). Then, the moment generating function $M(t)$ of the random variable $\xi$ is given by

$$
M(t)=E_{s}\left[e^{\xi t}\right]=\sum_{n=0}^{\infty} E_{s}\left[\xi^{n}\right] \frac{t^{n}}{n !},
$$

where the moments $E_{s}\left[\xi^{n}\right]$ of order $n$ are given by

$$
E_{s}\left[\xi^{n}\right]=\sum_{k=0}^{n} \frac{n !}{(n-k) !} \frac{\Gamma(s-k)}{\Gamma(s)} \frac{\Phi_{\lambda, \mu ; \nu}(z, s-k, 1 ; p)}{\Phi_{\lambda, \mu ; \nu}(z, s, 1 ; p)} .
$$

Proof. The assertion in (5.2) can be derived easily by using the series expansion of $e^{\xi t}$. To establish (5.3), we observe that

$$
\frac{d}{d a}\left\{\Phi_{\lambda, \mu ; \nu}(z, s, a ; p)\right\}=-s \Phi_{\lambda, \mu ; \nu}(z, s+1, a ; p),
$$

which follows readily from $(2.1)$, and thus from the definition of $E_{s}\left[\xi^{n}\right]$, we have

$$
\begin{aligned}
E_{s}\left[\xi^{n}\right]=\int_{1}^{\infty} a^{n} f_{\xi}(a) d a & \\
& =\frac{s}{\Phi_{\lambda, \mu ; \nu}(z, s, 1 ; p)} \int_{1}^{\infty} a^{n} \Phi_{\lambda, \mu ; \nu}(z, s+1, a ; p) d a \\
& =-\frac{1}{\Phi_{\lambda, \mu ; \nu}(z, s, 1 ; p)} \int_{1}^{\infty} a^{n} \frac{d}{d a}\left\{\Phi_{\lambda, \mu ; \nu}(z, s, a ; p)\right\} d a \\
& =\left[-\frac{a^{n} \Phi_{\lambda, \mu ; \nu}(z, s, a ; p)}{\Phi_{\lambda, \mu ; \nu}(z, s, 1 ; p)}\right]_{a=1}^{\infty}+ \\
& \quad+\frac{n}{\Phi_{\lambda, \mu ; \nu}(z, s, 1 ; p)} \int_{1}^{\infty} a^{n-1} \Phi_{\lambda, \mu ; \nu}(z, s, a ; p) d a \\
& =1-\frac{\lim _{a \rightarrow \infty}\left\{\frac{a^{n} \Phi_{\lambda, \mu ; \nu}(z, s, a ; p)}{\Phi_{\lambda, \mu ; \nu}(z, s, 1 ; p)}\right\}+}{\Phi_{\lambda, \mu ; \nu}(z, s, 1 ; p)} \int_{1}^{\infty} a^{n-1} \Phi_{\lambda, \mu ; \nu}(z, s, a ; p) d a \\
& =1+\frac{n}{\Phi_{\lambda, \mu ; \nu}(z, s, 1 ; p)} \int_{1}^{\infty} a^{n-1} \Phi_{\lambda, \mu ; \nu}(z, s, a ; p) d a,
\end{aligned}
$$


where, in addition to the derivative property (5.4), we have used the following limit formula:

$$
\begin{aligned}
& \lim _{a \rightarrow \infty}\left\{a^{n} \Phi_{\lambda, \mu ; \nu}(z, s, a ; p)\right\}=\lim _{a \rightarrow \infty}\left\{\frac{a^{n}}{\Gamma(s)} \int_{0}^{\infty} t^{s-1} e^{-a t} F_{p}\left(\lambda, \mu ; \nu ; z e^{-t}\right) d t\right\} \\
& \quad=\frac{1}{\Gamma(s)} \int_{0}^{\infty} t^{s-1} \lim _{a \rightarrow \infty}\left\{a^{n} e^{-a t}\right\} F_{p}\left(\lambda, \mu ; \nu ; z e^{-t}\right) d t \\
& =0
\end{aligned}
$$

Consequently, we have the following reduction formula for $E_{s}\left[\xi^{n}\right]$ :

$$
E_{s}\left[\xi^{n}\right]=1+\frac{\Phi_{\lambda, \mu ; \nu}(z, s-1,1 ; p)}{\Phi_{\lambda, \mu ; \nu}(z, s, 1 ; p)} \frac{n}{s-1} E_{s-1}\left[\xi^{n-1}\right],
$$

and by iterating the recurrence (5.5), we arrive at the desired result (5.3).

\section{References}

[1] M. A. Chaudhry, A. Qadir, M. Raflque, and S.M. Zubair, Extension of Euler's Beta function, J. Comput. Appl. Math., 78, (1997), 19-32

[2] M. A. Chaudhry, A. Qadir, H. M. Srivastava, and R. B. Paris, Extended hypergeometric and confluent hypergeometric functions, Appl. Math. Comput., 159, (2004), 589-602

[3] M. A. Chaudhry and S. M. Zubair, On a Class of Incomplete Gamma Functions with Applications, Chapman and Hall, (CRC Press Company), Boca Raton, London, New York and Washington, D. C., 2001

[4] J. Choi, D. S. Jang, and H. M. Srivastava, A generalization of the Hurwitz-Lerch Zeta function, Integral Transforms Spec. Funct., 19, (2008), 65-79

[5] A. Erdélyi, W. Magnus, F. Oberhettinger, and F. G. Tricomi, Higher Transcendental Functions, McGraw-Hill Book Company, New York, Toronto and London, I, 1953

[6] A. Erdélyi, W. Magnus, F. Oberhettinger, and F. G. Tricomi, Higher Transcendental Functions, McGraw-Hill Book Company, New York, Toronto and London, II, 1955

[7] M. Garg, K. Jain, and S. L. Kalla, A further study of general Hurwitz-Lerch zeta function, Algebras Groups Geom., 25, (2008), 311-319

[8] S. P. Goyal and R. K. Laddha, On the generalized Zeta function and the generalized Lambert function, Ganita Sandesh, 11, (1997), 99-108 
[9] S. D. Lin and H. M. Srivastava, Some families of the Hurwitz-Lerch Zeta functions and associated fractional derivative and other integral representations, Applied Mathematics and Computation, 154, (2004), 725-733

[10] M.A. Özarslan and E. Özergin, Some generating relations for extended hypergeometric function via generalized fractional derivative operator, Math. Comput. Modelling, 52, (2010), 1825-1833

[11] H. M. Srivastava and J. Choi, Series Associated with the Zeta and Related Functions, Kluwer, Acedemic Publishers, Dordrecht, Boston and London, 2001

[12] H. M. Srivastava and J. Choi, Zeta and q-Zeta Functions and Associated Series and Integrals, Elsevier Science, Publishers, Amsterdam, London and New York, 2012

[13] H. M. Srivastava, R.K. Saxena, T.K. Pogány, and Ravi Saxena, Integral and computational representations of the extended Hurwitz-Lerch Zeta function, Integral Transforms Spec. Funct., 22, (2011), 487-506

[14] E. T. Whittaker and G. N. Watson, A Course of Modern Analysis: An Introduction to the General Theory of Infinite Processes and of Analytic Functions ; With an Account of the Principal Transcendental Functions,Fourth edition, Cambridge University Press, Cambridge, London and New York, 1963

Rakesh K. Parmar

Department of Mathematics

Government College of Engineering and Technology

Bikaner-334004

Rajasthan State

India

E-mail: rakeshparmar27@gmail.com

R. K. Raina

Department of Mathematics

M.P. University of Agriculture and Technology

Udaipur 313002

Rajasthan State, India

Present address: 10/11 Ganpati Vihar, Opposite Sector 5,

Udaipur- 313002, Rajasthan State, India

E-mail: rkraina_7@hotmail.com

Received: 5.11.2014

Accepted: 29.11.2014 